Revue internationale P.M.E.

Économie et gestion de la petite et moyenne entreprise

\title{
Comportement et gouvernance des moyennes entreprises familiales au capital ouvert
}

\section{Fabrice Le Vigoureux et Pascal Aurégan}

Volume 23, numéro 3-4, 2010

URI : https://id.erudit.org/iderudit/1012494ar

DOI : https://doi.org/10.7202/1012494ar

Aller au sommaire du numéro

Éditeur(s)

Presses de l’Université du Québec

ISSN

0776-5436 (imprimé)

1918-9699 (numérique)

Découvrir la revue

Citer cet article

Le Vigoureux, F. \& Aurégan, P. (2010). Comportement et gouvernance des moyennes entreprises familiales au capital ouvert. Revue internationale P.M.E., 23(3-4), 71-93. https://doi.org/10.7202/1012494ar
Résumé de l'article

Les moyennes entreprises familiales au capital ouvert, ou adossées, méritent d'être mieux connues et mieux étudiées. Leur comportement stratégique, le plus souvent entrepreneurial, semble pouvoir être expliqué par une structure de propriété particulière, qui combine culture familiale et compétences " externes » issues d'un actionnariat minoritaire impliqué (capital-risque, capital-investissement, groupe financier ou industriel). Cet actionnariat minoritaire irrigue et modifie l'organisation et les orientations de l'entreprise. L'approche cognitive de la gouvernance valorise d'ailleurs ces apports extérieurs comme autant de nouveaux leviers ou de réseaux en faveur de l'innovation en relativisant la dimension disciplinaire des dispositifs de gouvernance. 


\title{
Comportement et gouvernance des moyennes entreprises familiales au capital ouvert
}

Fabrice LE VIGOUREUX

Pascal AURÉGAN

Université de Caen Basse-Normandie, France

MOTS CLÉS

Moyenne entreprise-Entreprise familiale-

Approche cognitive de la gouvernance

\begin{abstract}
LES AUTEURS
FABRICE LE VIGOUREUX, est maître de conférences habilité à diriger des recherches à l'IAE de Caen Basse-Normandie, dont il assume la direction. II poursuit ses travaux, au sein du laboratoire NIMEC, sur la finance comportementale et les moyennes entreprises. Adresse: IAE de Caen-3, rue Claude-Bloch, B.P. 5160-14075 Caen Cedex. Courriel: <fabrice. levigoureux@unicaen.fr>.

PASCAL AURÉGAN est maître de conférences habilité à diriger des recherches à l'IAE de Caen Basse-Normandie et chercheur au laboratoire NIMEC. Ses recherches portent, d'une part, sur l'influence des perceptions temporelles sur les comportements stratégiques des dirigeants et, d'autre part, sur les liens et interactions entre projets et management stratégique. Adresse: IAE de Caen, 3, rue Claude-Bloch, B.P. 5160, 14075 Caen Cedex. Courriel :<pascal. auregan@unicaen.fr>.
\end{abstract}

\section{RÉSUMÉ}

Les moyennes entreprises familiales au capital ouvert, ou adossées, méritent d'être mieux connues et mieux étudiées. Leur comportement stratégique, le plus souvent entrepreneurial, semble pouvoir être expliqué par une structure de propriété particulière, qui combine culture familiale et compétences "externes " issues d'un actionnariat minoritaire impliqué (capital-risque, capital-investissement, groupe financier ou industriel). Cet actionnariat minoritaire irrigue et modifie l'organisation et les orientations de l'entreprise. L'approche cognitive de la gouvernance valorise d'ailleurs ces apports extérieurs comme autant de nouveaux leviers ou de réseaux en faveur de l'innovation en relativisant la dimension disciplinaire des dispositifs de gouvernance. 


\begin{abstract}
Medium-sized public or secured family enterprises deserve to be better analyzed and understood. Their often entrepreneurial strategic behaviour may be explained through a specific ownership structure, one which comprises family culture and external expertise, stemming from participant minority shareholding (venture capital, investment capital, financial holding group or industrial group). This minority partnership helps channel and modify business organizations and orientations. Moreover, this cognitive governance method lends value to these external contributors as new levers and networks promoting innovation by helping put the disciplinary dimensions of governance mechanisms into perspective.
\end{abstract}

\title{
RESUMEN
}

Las medianas empresas familiares de capitales variables o respaldadas por capital riesgo, deberían ser mejor conocidas y estudiadas. Su comportamiento estratégico, frecuentemente de tipo empresarial, se revela como una estructura de propiedad particular que combina la cultura familiar con competencias «externas " resultantes de una participación minoritaria involucrada (capital riesgo, capital de inversión, grupo financiero o grupo industrial). Esta participación minoritaria riega y modifica la organización y la dirección de la empresa. El enfoque cognitivo de la gobernabilidad privilegia estos aportes externos que constituyen nuevos propulsores o redes para la innovación poniendo en perspectiva la dimensión disciplinaria de los dispositivos de gobernabilidad.

\section{ZUSAMMENFASSUNG}

Mittelständische Familienunternehmen mit offener Kapitalstruktur oder angelehnte Familienunternehmen sollten verstärkt bekannt und untersucht werden. Ihr strategisches, häufig unternehmerisches Vorgehen scheint durch eine besondere Eigentumsstruktur begründet zu sein, die Familienkultur und „externe“, von beteiligten Minderheitsaktionären bereitgestellte Kompetenzen (Wagniskapital, Risikokapital, Finanz-oder Industriekonzern) verbindet. Diese Minderheitsaktionäre nähren und verändern die Organisation und die Ausrichtungen des Unternehmens. Der kognitive Ansatz der Unternehmensführung valorisiert im Übrigen diese externen Beiträge als jeweils neue innovationsfördernde Faktoren oder Netze und relativiert dabei die disziplinarische Dimension der Unternehmensführungsinstrumente.

\section{Introduction}

Les modèles de développement de l'entreprise montrent qu'au-delà d'une certaine taille, une direction par délégation se met en place, l'organisation se décentralise et se complexifie. De même, avec la taille, la propriété a tendance à se morceler et à se dissocier de la gestion (ce que le langage conceptuel de la théorie des droits de propriété traduit par une «atténuation » de la fonction de décision). Ainsi, à la différence de la petite entreprise dans laquelle 
les grandes fonctions de la gestion sont assumées ou étroitement contrôlées par un dirigeant-propriétaire, la moyenne entreprise se définit souvent dans la littérature comme une organisation structurée, dotée d'un encadrement intermédiaire, s'appuyant sur un actionnariat bien identifié, parfois fractionné (sans pour autant être diffus) et sans lien systématique avec la fonction managériale (GREPME, 1994; Le Vigoureux, 1997).

S'inscrivent dans le périmètre de cette définition aussi bien les filiales de groupes de taille moyenne (dont le poids relatif augmente sur les dernières décennies) que les entreprises strictement patrimoniales sans lien de dépendance en capital avec une autre unité économique. Entre ces deux formes extrêmes de structures d'actionnariat, une troisième, rarement traitée comme catégorie à part entière, peut être isolée au sein de ce qu'il est convenu d'appeler le «tissu intermédiaire»: la moyenne entreprise «adossée», souvent familiale, au capital ouvert à des entités minoritaires (capital-investisseur, groupe, acteur institutionnel). Les évolutions démographiques récentes du tissu économique invitent à porter un regard particulier sur ce type d'entreprise. La structuration de l'offre de capital-investissement, la clarification du positionnement stratégique des plus grandes unités (les conduisant au désengagement ou à l'abandon d'activités périphériques), ou encore les pratiques d'essaimage alimentent en effet le marché des reprises de parts sociales non cotées et contribuent à l'apparition de nouvelles entreprises adossées ou à l'émergence de nouvelles opportunités d'adossement pour les entreprises familiales indépendantes ${ }^{1}$.

L'apparition ou l'existence d'une entité externe minoritaire dans le capital d'une entreprise familiale n'est pas neutre. Plusieurs travaux, mentionnés plus loin dans cet article, montrent qu'elle impacte substantiellement le comportement stratégique, l'organisation et la gouvernance de l'entreprise, en lui conférant un caractère plus entrepreneurial et plus «professionnel». L'ouverture du capital, même très partielle, n'apparaît pourtant pas toujours comme une trajectoire «naturelle». L'une des manifestations les plus visibles des résistances des entreprises patrimoniales à la perte partielle d'indépendance réside dans l'utilisation hiérarchisée des sources de financement, conformément à la théorie du pecking-order (Myers, 1984) dont le pouvoir

1. La qualité d'entreprise adossée s'acquiert en effet selon deux cheminements types:

- l'ouverture partielle du capital d'une entreprise strictement patrimoniale à une entité externe (autre entreprise, société de capital-investissement, institutionnels), à travers une vente de ses parts ou actions ou une souscription de parts ou d'actions nouvelles créées dans le cadre d'une augmentation de capital;

- la cession d'une entreprise à une ou plusieurs personnes physiques qui, constituant un tour de table pour le rachat, intègrent une ou plusieurs sociétés dans le capital du cédant. La coalition de personnes physiques détient le contrôle de l'entreprise. 
explicatif se révèle fort dans le cas des entreprises familiales, des PME et des sociétés fermées (Norton, 1991; Berger et Udell, 1998; Poutziouris, 2001). L'ouverture du capital est souvent présentée comme un «financement de dernier ressort», lorsque l'autofinancement et la capacité d'endettement apparaissent insuffisants pour répondre à une opportunité d'investissement. Les propriétaires des entreprises familiales, plus encore lorsqu'ils sont fondateurs et/ou lorsqu'ils assument la fonction de direction, peuvent en effet être amenés à développer au cours du temps une culture de l'indépendance, à confondre leur destin à celui de l'entreprise et à adopter des attitudes de protection envers un territoire qu'ils estiment avoir largement construit. La perspective d'ouverture du capital engendre un certain nombre de craintes qui peuvent les conduire à privilégier la non-croissance aux dépens du maintien ou de l'amélioration de la position concurrentielle.

L'identité familiale et la confiance qu'une entreprise familiale suscite auprès de ses parties prenantes en sont-elles pour autant réellement affectées lorsqu'elle choisit d'ouvrir partiellement son capital? La relation d'agence introduite par la coexistence de deux pôles d'actionnaires de nature différente est-elle susceptible d'altérer les forces et l'objectif de pérennité habituellement relevés pour une entreprise familiale «indépendante»? Cet article tend à répondre par la négative à ces questions et soutient l'hypothèse qu'un actionnariat minoritaire extérieur à la famille constitue bien souvent la source d'un renouveau entrepreneurial, limitant les risques de sclérose, et peut être considéré, à l'appui notamment des approches cognitives de la gouvernance, comme un levier pour l'innovation, l'introduction d'idées nouvelles et l'ouverture à de nouveaux réseaux relationnels.

Cet article se nourrit d'une littérature centrée sur l'entreprise familiale, rappelée dans ses grandes lignes dans un premier point, mettant en exergue ses qualités intrinsèques et ses fragilités (1). Il cherche ensuite à identifier, pour les entreprises familiales au capital initialement fermé, certains effets d'ordre stratégique ou organisationnel de l'adossement à un actionnariat externe minoritaire ou d'une gouvernance ouverte, que traduit notamment une propension plus forte au changement stratégique et à l'innovation (2). Les récentes approches cognitives de la gouvernance rappelées dans le troisième point éclairent d'un jour nouveau le caractère plus entrepreneurial des moyennes entreprises familiales adossées et permettent de mieux en saisir certaines de leurs caractéristiques (3). 


\section{Qualités intrinsèques et fragilités des entreprises familiales dans la littérature}

Les entreprises familiales ont fait l'objet, sur les deux dernières décennies, d'une importante littérature. Un certain nombre d'enquêtes ou d'études d'obédience professionnelle (par exemple KPMG, 2007) ou académique (Arrègle et Mari, 2010) pointent les performances souvent supérieures des entreprises familiales comparativement aux entreprises non familiales, sur des critères stratégiques, sociaux, financiers ou organisationnels. Allouche, Amann et Garaudel (2007) recensent et articulent la littérature relative aux explications liées à la surperformance des entreprises familiales. Deux cadres théoriques majeurs sont mobilisés: d'une part, le courant contractualiste, pour lequel la réduction des coûts de contrôle et d'incitation des dirigeants non familiaux et la nature «patiente» de l'actionnariat fournissent les explications les plus convaincantes; d'autre part, le courant néo-institutionnaliste, qui s'appuie sur l'homogénéité du système de valeurs des entreprises familiales et l'imbrication des systèmes sociaux famille-entreprise.

Parmi les explications prenant comme cadre l'approche contractualiste (principalement la théorie de l'agence), la convergence ou la confusion des intérêts entre direction, actionnariat et administrateurs limite ou rend moins probable l'apparition de coûts de surveillance, d'engagement ou d'opportunité liés à l'opportunisme précontractuel (sélection adverse) ou postcontractuel (aléa moral) d'agents économiques rationnels. La surperformance trouverait donc son origine dans des coûts d'agence nuls ou marginaux. De plus, l'orientation à long terme des actionnaires familiaux, la durée importante des mandats managériaux, l'importance accordée aux objectifs de pérennité de l'entreprise et du contrôle familial (Mignon, 2000) limiteraient toute décision court-termiste inefficiente que l'on rencontre dans le contexte de la firme managériale où le dirigeant doit être «discipliné», car il est par nature négligent, engage des dépenses discrétionnaires, cherche à accroître son prestige en privilégiant la taille de l'entreprise plutôt que sa rentabilité, etc.

La manifestation sans doute la plus visible de cette «préférence» pour le long terme se trouve dans les études mettant en évidence le moindre recours à l'endettement (Allouche et Amann, 1998), la part importante du réinvestissement des résultats et la faiblesse corrélative de la politique de distribution de dividendes, que l'entreprise soit cotée (Calvi-Reveyron, 2000) ou non (Hirigoyen, 1984). Pour Kenyon-Rouvinez et Ward (2004,p. 50), «cette politique d'actionnariat est le pilier de la réussite à long terme de l'entreprise familiale car elle permet l'apport de capitaux à un moindre coût, détenus par 
les membres de la famille qui ne demandent pas un retour sur investissement rapide, mais plutôt une augmentation sur le long terme de la valeur de l'entreprise (patient capital)».

La vision néo-institutionnaliste de l'entreprise fournit un deuxième ensemble d'explications à la meilleure performance des entreprises familiales. La famille constitue ici un réseau social clanique offrant une régulation efficiente des transactions interindividuelles qui se forment sur des bases de loyauté et sur un système de valeurs homogène ${ }^{2}$. Ce qui est ici valorisé n'est pas tant la faiblesse des coûts d'agence liée à la confusion des fonctions managériales et actionnariales qu'un fonctionnement assis sur la confiance (Allouche et Amann, 1998), la solidarité et l'altruisme des acteurs clés de l'entreprise. Cette confiance alimente des décisions inscrites sur le long terme et sécurise les arbitrages en faveur d'une ligne stratégique durable en limitant les questionnements conjoncturels sur la portée immédiate des décisions.

Dans ce même corpus explicatif, en référence à une approche par les ressources et les compétences, certains auteurs ont développé le concept de «familiness» ou «familiarisme» désignant les ressources uniques qu'une entreprise possède du fait des interactions entre la famille, ses membres et l'activité de l'entreprise (Habbershon et Williams, 1999). Les valeurs inhérentes à la famille, la bonne volonté, l'engagement et l'enthousiasme de leurs membres, la valorisation d'un comportement conforme à l'éthique, la perception de l'entreprise par les parties prenantes ou stakeholders comme étant digne de confiance, le sentiment puissant d'appartenance, une information mieux partagée et de meilleure qualité sont autant d'éléments qui donnent à l'entreprise familiale des ressources cognitives distinctives (Sirmon et Hitt, 2003; Anderson et Reeb, 2003; Arrègle, Durand et Very, 2004; Zellweger, Eddleston et Kellermanns, 2010). Ces éléments se retrouvent également dans les approches fondées sur la théorie de l'intendance (stewardship theory) ${ }^{3}$ appliquée aux entreprises familiales: Miller, Breton-Miller et Scholnick (2008) défendent à l'appui de comparaisons empiriques ces approches présentant les propriétaires d'entreprises familiales comme profondément attentifs

2. De manière extrême, Miller, coauteur d'une étude Barclays (2009) réalisée sur la base d'un sondage auprès de clients de la gestion de fortune de la banque britannique, relève que les familles religieuses ont tendance à avoir des entreprises plus performantes.

3. Pour la «stewardship theory» ou théorie de l'intendance, les intérêts des dirigeants et des actionnaires sont alignés et sont mus par des ressorts communs, le sens du devoir et non l'égoïsme ou le gain individuel.Ainsi, dans les entreprises familiales, la confusion ou la proximité des fonctions de décision, de propriété et de contrôle facilitent les comportements d'intendance (Davis, Shoorman et Donaldson, 1997). 
à la «continuité» de l'entreprise, attachés à favoriser la «communauté» des employés et à nourrir des relations loyales et durables avec leurs clients («connexion»).

Ces explications positives sur la solidité des entreprises familiales font toutefois l'objet de nuances ou de critiques par d'autres études qui relèvent certaines fragilités ou inclinations sous-optimales. Ces fragilités sont de deux ordres principaux: des orientations conservatrices ne permettant pas de saisir tout le potentiel de croissance et un enracinement négatif du dirigeant favorisant l'émergence de coûts d'agence d'un type nouveau (intrafamilial), de situations conflictuelles ou d'altération des liens «fraternels».

Dans l'introduction de cet article, pour expliquer la difficile trajectoire d'adossement d'une entreprise au capital fermé, nous évoquions une certaine rationalité de la «non-croissance». L'une des premières caractéristiques de l'actionnariat familial étant que l'entreprise constitue généralement l'un des principaux biens de la famille, cela peut l'amener à adopter une gestion moins risquée en matière d'investissement, de financement ou d'innovation et limiter à l'évidence la saisie d'opportunités de croissance qu'un financement actionnarial externe permettrait d'envisager. Ainsi, l'excès de prudence ou de conservatisme des entreprises familiales, fréquemment relevé (Donckels et Fröhlich, 1991; Sharma, Chrisman et Chua, 1997), peut amener à des mouvements stratégiques limités à des marchés étroits, à la croissance faible, voire à la disparition prématurée ${ }^{4}$ ( $c f$. Miller, Breton-Miller et Scholnick, 2008). Pour Mignon (2009), le poids des expériences passées, la focalisation des ressources sur un seul domaine d'activité peuvent conduire à une certaine myopie stratégique, aggravée par le sentiment d'invulnérabilité engendré par une ancienneté importante.

La deuxième grande faiblesse pointée par la littérature sur les entreprises familiales est liée à l'apparition possible, après une certaine durée de vie, de phénomènes d'enracinement négatif ou de népotisme générateurs de conflits ${ }^{5}$. L'âge semble être dans plusieurs études une variable explicative

4. Miller et al. (2008) décrivent, en opposition à la perspective «stewardship» dans laquelle ils s'insèrent, la perspective «stagnation" pointant le manque de ressources, le caractère conservateur, la croissance faible et la courte durée de vie des entreprises familiales.

5. Conflits recensés par une enquête sur les entreprises familiales françaises, publiée en 2006 par PriceWaterhouseCoopers, et réalisée auprès de 102 entreprises couvrant plusieurs secteurs d'activité. Selon cette étude, seuls $5 \%$ des dirigeants interrogés ne mentionnent pas de sources de conflits. Les dissensions citées par les $95 \%$ restants sont relatives, de façon presque équivalente, à la constitution des équipes de direction et de contrôle, à l'influence des membres de la famille, aux questions financières et à la transmission. 
de l'émergence de ce type de phénomène (Morck, Shleifer et Vishny, 1988). Pour Gomez-Mejia, Nunez-Nickel et Gutierrez (2001), les conséquences de l'enracinement du dirigeant peuvent même être plus sérieuses dans les entreprises familiales que dans les entreprises non familiales.

Hirigoyen (2002 et 2008, p. 1902) observe ainsi que «les liens familiaux évoluent au cours du cycle de vie et affectent l'entreprise à travers des conflits d'intérêt significatifs, une information et un altruisme asymétriques spécifiques entre les membres de la famille». L'auteur pointe l'altération graduelle, au cours du cycle de vie de l'entreprise familiale, des liens entre les membres de la famille. Dans les périodes de succession, l'entreprise familiale affronte des changements dans les «patterns» des liens familiaux qui atténuent le degré de la confiance mutuelle. Si les coûts de surveillance ou de contrôle sont moins élevés dans une entreprise familiale, des coûts de rivalités fraternelles ou liés à des comportements autocratiques ou népotiques peuvent émerger (les études de cas d'entreprises familiales menées par Kets de Vries et publiées en 1988 en fournissent des illustrations). Certains membres des générations suivantes préfèrent parfois récupérer leur part plutôt que de la voir immobilisée dans une affaire à laquelle ils ne sont pas directement associés.

La famille ne peut donc pas toujours être considérée comme une entité stable et cohésive. Avec les générations, le caractère «dynastique» de la famille s'accroît et les liens sont susceptibles de s'affaiblir entre ses membres. Lorsque la famille devient ce que d'aucuns appellent un «consortium de cousins», les conflits potentiels s'accroissent, les systèmes informels de contrôle mutuel perdent 1) en efficacité et les branches de la famille écartées du premier cercle de pouvoir peuvent s'estimer lésées par le noyau familial dominant, 2) en capacité à renoncer à des investissements pertinents jugés unilatéralement trop risqués ou obligeant à acquérir de nouvelles compétences (Schulze, Lubatkin et Dino, 2001 et 2002).

Le népotisme, la captation des postes de direction par des managers inexpérimentés ou moins performants que ce que peut fournir le marché des dirigeants (certes inefficient) et en général les coûts liés à l'altruisme (sélectif) de la figure familiale dominante peuvent être considérés comme des composantes de coûts d'agence d'un genre nouveau dans l'entreprise familiale. 


\section{Les moyennes entreprises adossées: apports des minoritaires et vertus d'une gouvernance «métissée »}

L'exposé synthétique des principales forces et fragilités des entreprises familiales interroge la capacité de celles ayant procédé à une ouverture partielle de leur capital ou de leur gouvernance à préserver ou consolider leurs forces (continuité, esprit communautaire, «familiness», etc.) en contournant les perspectives les moins flatteuses (népotisme, conflits intrafamiliaux, conservatisme stratégique, etc.). L'hypothèse se construit à l'aune de nombreux travaux montrant les bénéfices d'une gouvernance ouverte sur les orientations stratégiques ou les dimensions organisationnelles des entreprises familiales.

Plusieurs auteurs (par exemple Fernandez et Cobas, 1998; Amit, Brander et Zott, 1998; Stephany, 2001; Gabrielsson et Huse, 2002) ont montré que l'adossement d'une entreprise à une structure de capital-investissement ou de capital-risque s'accompagne de la mise en place d'un contrôle contractualisé pouvant impliquer des changements organisationnels significatifs au sein de l'entreprise (définition d'une fonction financière et d'un contrôle de gestion, émergence de tableaux de bord, etc.). Pour ces auteurs, l'apparition d'une entité externe dans le capital d'une entreprise permet certes l'apport de ressources financières nouvelles, mais également des apports résiliaires, techniques, financiers, commerciaux, parfois informels ou indirects. L'actionnaire extérieur peut jouer le rôle de garant du potentiel de l'entreprise et, ce faisant, signaler sa qualité, accroître sa réputation et lui permettre ainsi d'accéder à des ressources financières qui, en son absence, lui auraient été inaccessibles ${ }^{6}$. Le tableau 1, établi par J. Pouget et E. Stephany (2002), synthétise par exemple l'impact de l'entrée d'un capital-risqueur sur les principales fonctions de l'entreprise.

L'adossement à un groupe peut avoir certains effets similaires. Ciéply (1995) montre en particulier que l'établissement d'un partenariat financier entre une entreprise «établie» et une entreprise «non établie» exerce un effet de levier sur la capacité d'endettement de cette dernière en réduisant les incertitudes et l'asymétrie d'information entre l'entreprise et ses partenaires bancaires. Un établissement bancaire peut obtenir des informations sur une entreprise non établie en observant les interactions de cette entreprise avec une grande entreprise réputée ayant un bon rating auprès des bailleurs de

6. On relèvera toutefois des approches soulignant aussi l'émergence de conflits et d'incompréhensions entre capital-investisseurs et dirigeants dans les travaux de Guéry-Stevenot (2006). 


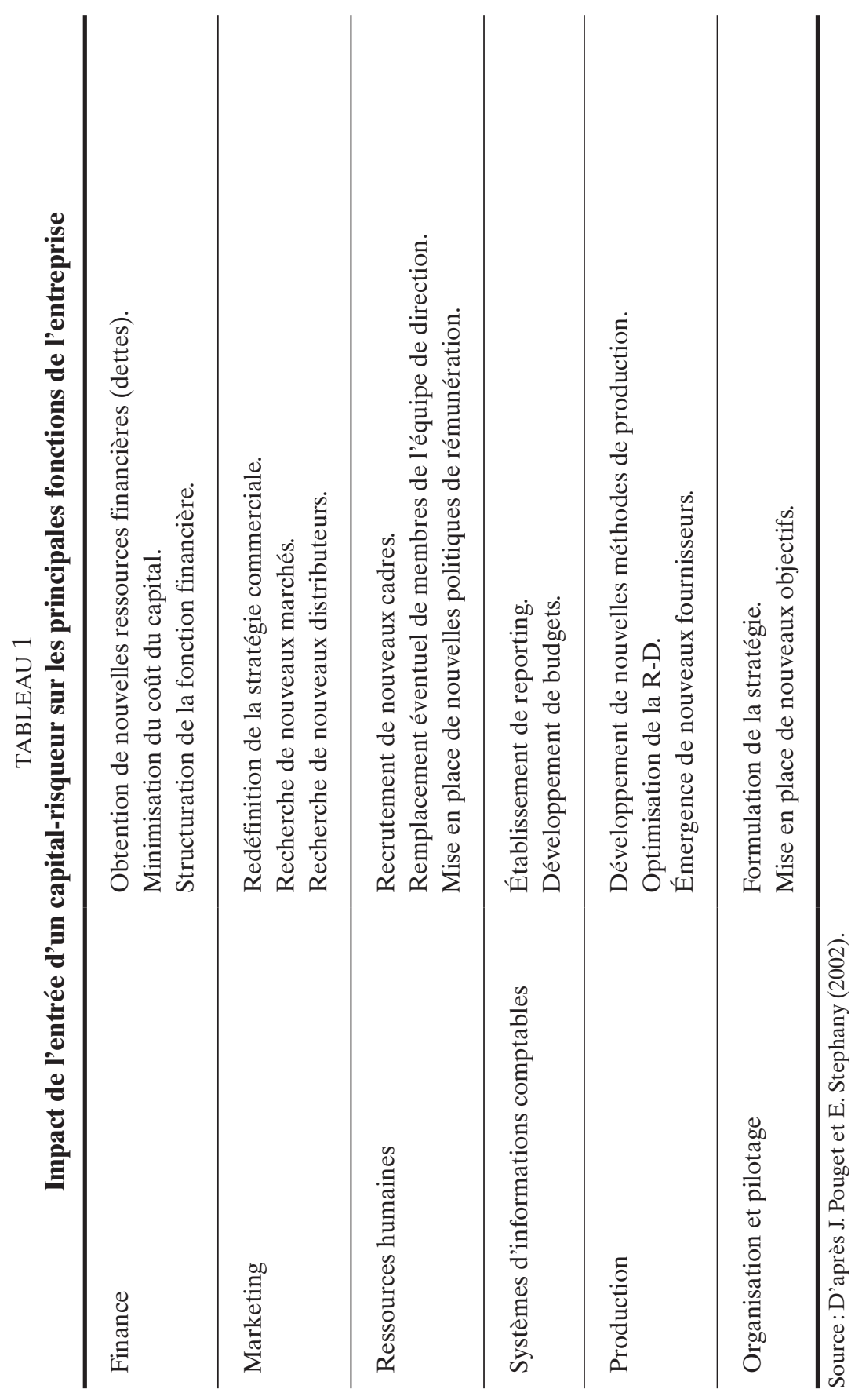


fonds. Cette grande entreprise est une interface qui modifie les conditions d'accès au financement bancaire d'un partenaire se situant hors des normes de la communauté financière.

Les effets d'un capital ouvert sur l'innovation peuvent être également soulignés. Les moyennes entreprises familiales adossées, comparées à leurs homologues indépendantes ou aux filiales de taille moyenne, laissent apparaître des comportements stratégiques entrepreneuriaux, proactifs, caractérisés par des politiques d'innovation technologique et/ou de produit importante et une faible aversion pour le risque (Le Vigoureux, 1997). Une étude du Centre d'analyse stratégique (2009) relève aussi que l'appartenance à un groupe est un facteur contribuant à accroître la propension à innover des entreprises. Ce constat corrobore les analyses de Stephany (2003) et de Couderc et Stephany (2008), selon lesquelles une gouvernance ouverte, caractérisée par la présence d'actionnaires extérieurs ou incluant des acteurs internes et externes à l'entreprise, favorise les dynamiques d'innovation. Sur ce point, l'hypothèse retenue par Cosier et Harvey (1998) est que le conflit d'intérêt inhérent par exemple à la distinction entre les acteurs qui dirigent et ceux qui contrôlent est source d'un plus grand potentiel de coopération et introduit une tension organisationnelle nécessaire au développement de la création, de l'innovation et favorisant de meilleures performances? ${ }^{7}$.

L'influence d'une gouvernance ouverte sur des variables clés de la stratégie de l'entreprise est également relevée dans plusieurs études très significatives. Naldi et Nordqvist (2008) montrent ainsi pour les entreprises familiales que le degré d'ouverture de la structure de gouvernance impacte leur degré d'internationalisation ${ }^{8}$. Les auteurs mobilisent la théorie de la

7. Nous revenons sur cette notion de conflit dans la troisième partie de l'article. Notons à ce stade que pour plusieurs auteurs (par exemple Gallo et Vilaseca, 1998; Barth, Gulbrandsen et Schone, 2005; Charlier et Lambert, 2008), la séparation des fonctions de propriété et de management augmente la performance. L'étude de Charlier et Lambert (2008) construite sur la base d'un échantillon européen de 418 PME familiales montre ainsi que les entreprises familiales contrôlées et dirigées par la famille sont moins performantes que deux autres modes plus éclatés de gouvernance familiale: les entreprises contrôlées par la famille avec un dirigeant extérieur et, de manière plus nette encore, celles dirigées par une famille minoritaire (conformément à une hypothèse de Neubauer et Lank, 1998, et aux résultats d'Anderson et Reeb, 2003).

8. Les auteurs définissent une structure de gouvernance ouverte à partir des caractéristiques suivantes: des propriétaires externes, un dirigeant externe, des membres du conseil d'administration externes, une équipe de top management importante. Ils montrent que l'expansion sur les marchés étrangers (international scope) est favorisée par une ouverture sur toutes les dimensions de la structure de gouvernance. La pénétration de marchés étrangers (international scale) est favorisée par l'ouverture du niveau top management spécifiquement. 
dépendance sur les ressources, qu'ils jugent pertinente parce qu'elle permet d'appréhender des différences importantes de comportements stratégiques entre les entreprises familiales et la manière dont elles construisent des liens avec leurs environnements externes dans le but d'accéder à des ressources non familiales, essentielles à l'internationalisation. S'engager sur des marchés internationaux requiert une prise de risque considérable pour les propriétaires et les managers familiaux. L'internationalisation peut alors être freinée par la prudence, l'aversion au risque, le conservatisme, la résistance au changement des leaders familiaux et le manque de contrôle formel et de système de planification. Quand la famille surmonte sa peur de perdre le contrôle et ouvre la structure de gouvernance à des acteurs non familiaux, de nouvelles ressources extérieures à la famille et à la firme sont apportées facilitant l'internationalisation. Dans un registre similaire, Brunninge, Nordqvist et Wiklund (2007) montrent que le changement stratégique, pouvant passer par la diversification, l'innovation ou l'entrée sur de nouveaux marchés à l'international, est favorisé dans les PME aux structures de propriété ouvertes. Dans les entreprises étroitement contrôlées et fermées, la concentration des propriétaires au sein de la direction se traduit souvent pour ces auteurs par une plus grande aversion pour le risque, un moindre entrain, de moindres pressions ou incitations externes pour s'engager dans le changement ${ }^{9}$.

La présence d'administrateurs externes au sein du conseil d'administration traduit également une gouvernance «ouverte». Ces administrateurs, recrutés notamment pour leurs compétences particulières et leur regard extérieur, et qui n'exercent aucune fonction de direction et ne détiennent pas ou très peu d'actions de la société, semblent également influer sur la nature des orientations stratégiques de l'entreprise (Daily et Dalton, 1992; Mustakallio, Autio et Zahra, 2002). Pour Kenyon-Rouvinez et Ward (2004, p. 82), «l'expérience montre que les familles qui ont ouvert leur conseil à des membres indépendants réussissent à fixer des objectifs plus clairs, plus affinés, et sont plus performantes».

Ainsi, la présence d'une entité externe dans le capital de l'entreprise, la cohabitation d'acteurs d'origine ou de nature différente dans la propriété (directeurs ou administrateurs «externes») apparaissent associées à des impulsions organisationnelles nouvelles, modifiant les fonctions de l'entreprise, les dynamiques d'innovation, les orientations stratégiques ou même les performances. Certes, les coûts d'agence liés à la partition de la propriété ou à la séparation des fonctions de gouvernance sont plus importants que ceux

9. Si les entreprises fermées sont moins enclines au changement stratégique que celles présentant une gouvernance ouverte, les auteurs relèvent toutefois qu'un nombre important de personnes impliquées dans le management et la prise de décision dans la firme peut compenser, en partie, l'absence d'administrateurs externes. 
qui prévalent dans une moyenne entreprise familiale au capital fermé. Mais les effets décrits plus haut laissent présumer qu'un apport externe, qu'il concerne la propriété, la direction ou le contrôle, contribue à la revitalisation de l'entreprise, introduit un renouveau managérial et stratégique salvateur contrant les risques de conservatisme, de népotisme ou d'altruisme inefficient relevés dans les formes «pures » et anciennes des entreprises familiales. Suivant en cela Arrègle et Mari (2010, p. 100), on peut dire que «l'influence familiale» peut être préférable à l'exclusivité familiale. Comme le mentionnent ces auteurs, «l'influence familiale quand elle n'est pas associée à un contrôle total du capital a un impact positif sur les décisions et donc sur la performance de ces firmes. Elle permet aux autres parties prenantes de l'entreprise d'intervenir dans le processus de décisions stratégiques et d'équilibrer l'influence de la famille sur les décisions de l'entreprise» qui se retrouve alors «peu confrontée à des problèmes d'absence d'innovation ou de renouvellement, de pensée commune ou d'aliénation de certaines parties prenantes $»^{10}$.

Faut-il néanmoins voir dans ce «métissage» des fonctions de gouvernance, lorsqu'il s'installe dans une entreprise familiale, une altération de ses atouts spécifiques, une dislocation du système famille-entreprise, un morcellement de la confiance? La moyenne entreprise familiale adossée est-elle susceptible de perdre, du fait de son ouverture partielle à une entité externe, son «familiness»? Sans pouvoir être ici affirmatif ${ }^{11}$, notre hypothèse est celle d'une survivance active de la famille en cas d'adossement, gage de «continuité» (au sens de Miller, Breton-Miller et Scholnick, 2008) des relations commerciales et professionnelles. Les effets positifs de la culture familiale ont toutes les raisons de subsister car le contrôle des affaires, s'il n'est plus exclusif, demeure dans le domaine familial. La propriété comme la direction restent par essence le fait de (ou en étroite dépendance avec) l'actionnariat familial dominant. L'ouverture à un actionnariat externe est quant à elle un levier d'amplification du tissu relationnel de l'entreprise ou, selon les termes d'une récente étude sur les entreprises de taille intermédiaire en France, «un appui efficace et nécessaire au développement [...] par l'apport instantané de compétences surdimensionnées, bonnes pratiques, contacts et ouvertures [...] pour accompagner le changement d'état» (Rapport Retailleau, 2010, p. 86). Rien ne laisse penser qu'une ouverture partielle du capital puisse ainsi

10. On citera également Hirigoyen (2009, p. 404) qui s'appuie sur les travaux de Fueglistaller et Zellweger (2006) pour relever que la performance financière se dégrade lorsque l'influence de la famille est soit trop faible, soit trop forte.

11. La question est inscrite dans l'agenda de recherche des auteurs. 
altérer un héritage culturel familial patiemment construit ou que celui-ci ne puisse avec bénéfice se combiner à des compétences externes revitalisantes et à l'introduction de nouvelles modalités de gouvernance.

Ces modalités, qui ne se construisent pas seulement sur le terreau d'intérêts divergents ou de dispositifs « disciplinants », peuvent s'analyser comme des relations de coopération qui n'altèrent ni la continuité ni la confiance vis-à-vis de l'ensemble des parties prenantes. C'est le sens de l'approche cognitive de la gouvernance, récemment développée dans la littérature, et qui peut constituer un cadre explicatif intéressant de la propension des entreprises familiales adossées à innover, à s'internationaliser ou à oser le changement stratégique.

\section{L'approche cognitive de la gouvernance: cadre explicatif du caractère entrepreneurial de la moyenne entreprise adossée}

Les évolutions de l'analyse stratégique (Aurégan, Joffre et Le Vigoureux,1997), comme celles des fondements théoriques du gouvernement de l'entreprise, invitent à aborder les relations entre les différents partenaires de la firme comme des relations de coopération plutôt que d'agence et à adopter une vision de la firme qui valorise le rôle des ressources intangibles dans la création de valeur.

L'approche cognitive de la gouvernance, particulièrement promue dans l'espace francophone par Charreaux (2002a, 2002b, 2008) et Wirtz (2008a, 2008b), s'inscrit au confluent de différents courants comme la théorie comportementale de la firme, les théories de l'apprentissage organisationnel ou les théories des ressources et compétences. Le système de gouvernance se définit sur ces bases comme l'ensemble des mécanismes permettant d'avoir le meilleur potentiel de création de valeur en privilégiant les compétences, l'apprentissage, les capacités d'innovation.

Dans cette approche, la notion de conflit n'est pas envisagée de la même manière que dans l'approche disciplinaire classique de la gouvernance. Elle ne se réfère plus à la divergence d'intérêts des parties prenantes, liée à des objectifs différents et conduisant à la mise en place d'un système de surveillance ou d'incitation, mais à une incompréhension mutuelle susceptible d'être dépassée et potentiellement créatrice de valeur. Si l'excès de conflit cognitif doit bien sûr être évité, son absence est tout aussi dommageable car 
elle diminue la capacité à traiter de l'information complexe ${ }^{12}$. Les confrontations de points de vue, de visions et les discussions sur des bases raisonnées permettent d'élaborer des décisions mieux construites et plus appropriées que celles prises en l'absence de toute possibilité de dissonance cognitive initiale. Pour Forbes et Milliken (1999), la présence de désaccords ou de formulations critiques d'un conseil d'administration oblige le dirigeant à expliquer, justifier et possiblement modifier sa position en accueillant des perspectives alternatives sur des enjeux stratégiques importants. Pour ces auteurs, le conflit cognitif favorise la prise en compte d'alternatives plus nombreuses et leur évaluation plus attentive, ce qui contribue à la qualité de la prise de décision stratégique en environnement incertain. Le conseil n'est plus ici décrit seulement comme le support formel du lien actionnairesmanagers. Son rôle classique de contrôle ou de validation est étendu à un rôle de service, d'apprentissage mutuel, de mentoring, de renfort de compétences, de participation active à la formulation de la stratégie ${ }^{13}$. Comme le résume Wirtz (2008b, p. 105), «à l'inverse des conflits d'intérêts, les conflits cognitifs ne sont pas tous destructeurs de valeur, dans la mesure où la critique constructive peut avoir un impact positif sur l'émergence des projets stratégiques, suggérant des améliorations possibles, mettant en garde contre des dangers potentiels ou induisant même certaines innovations ».

L'actionnaire est ici envisagé, pour reprendre le titre d'un article de Charreaux (2002a), comme «un apporteur de ressources cognitives». Des trois composantes de la fonction entrepreneuriale, l'assomption du risque et de l'incertitude, la fonction managériale et la fonction «perceptive» associée au repérage des opportunités rentables, les deux dernières peuvent être qualifiées, selon l'auteur, de fonctions cognitives car liées à la vision et à la construction de connaissances. Dans cette approche, «ce qui fonde en premier l'efficience d'une firme, son aptitude à créer de la valeur, c'est sa capacité à réduire les conséquences des conflits cognitifs et à innover. Le fondement de l'efficience n'est plus de type allocatif et statique-la meilleure utilisation possible des ressources-mais de type productif, adaptatif et dynamique. L'objectif est d'assurer une croissance durable en particulier par la construction des opportunités de croissance» (Charreaux, 2002a, p. 103).

12. Le conflit cognitif, bien distinct du conflit d'intérêts, ne saurait non plus être confondu avec le conflit affectif. Comme le précise Marandon (2003), «le conflit cognitif concerne les tâches à accomplir et porte sur les objectifs et les modalités permettant de les atteindre, tandis que le conflit affectif est lié à la personnalité et émerge lorsque les enjeux associés aux tâches interfèrent avec les préférences, les opinions ou les principes moraux des personnes».

13. Ce qui était en germe, pour les PME, dans les travaux de Castaldi et Wortman en 1984. Voir à ce propos l'étude exploratoire sur les conseils d'administration dans les PME de Lofti et Wafa (2007). 
Les opportunités stratégiques ne sont plus appréhendées comme des données exogènes de l'environnement mais sont construites par les acteurs de la gouvernance en fonction de leurs connaissances et compétences spécifiques et de leurs interactions variées. La diversité des comportements est valorisée et l'innovation naît de la coexistence de schémas cognitifs différents au sein de l'entreprise. Les mécanismes de gouvernance ne cherchent pas en premier lieu à réduire les conflits d'intérêts, à aligner les intérêts des parties prenantes ou à contraindre le dirigeant de façon unilatérale en lui imposant une étroite discipline, mais sont appréhendés comme un levier à l'appui de l'action du dirigeant. Ces mécanismes ont ainsi un «pouvoir habilitant» (Wirtz, 2008a, p. 9), en soutenant, par exemple, une stratégie de forte croissance dans un contexte turbulent. Une bonne gouvernance cognitive repose dès lors sur la capacité du dirigeant à percevoir et construire de nouvelles opportunités, ainsi qu'à résorber les conflits cognitifs latents à ces choix stratégiques (Depret et Hamdouch, 2005; Charreaux, 2008).

Cette approche est particulièrement intéressante pour l'étude des entreprises familiales, et d'autant plus dans l'analyse de celles qui ont fait le pari de la croissance en ouvrant partiellement leur capital et/ou en privilégiant l'appui de compétences externes à la famille dans les organes de direction et de contrôle. Ces compétences externes ne cherchent pas en priorité à s'assurer de la probité de la coalition familiale dominante, car la valeur ne se crée pas par la seule discipline. Elles ont avant tout comme vocation, en s'appuyant par exemple sur leur connaissance intime des marchés et des technologies, à renforcer la base de connaissances stratégiques et managériales disponibles, à aider à la mise en œuvre des plans de développement, à apprendre à mieux gérer le processus entrepreneurial ou à soutenir la qualité et la rapidité de la prise de décision. Elles constituent ainsi des leviers particulièrement valorisés dans les entreprises en croissance. Les actionnaires externes à la famille peuvent ainsi concourir à une fonction exploratoire en apportant des savoirs, des compétences non disponibles au sein de la firme. Ils peuvent également guider ou conseiller les dirigeants vers l'acquisition de nouvelles compétences génératrices de valeur ou synergies, à travers notamment la recherche d'alliances ou des opérations de fusion ou d'acquisition (Charreaux et Wirtz, 2006).

La moyenne entreprise adossée peut donc ainsi être appréhendée non principalement comme le lieu où s'exercent des pouvoirs et contre-pouvoirs, mais comme une structure de propriété privilégiée pour voir s'épanouir des mécanismes de soutien à la formulation de la stratégie et à la conception des actions de sa mise en œuvre. Ces mécanismes de gouvernance, bien plus que d'aider à une simple sélection des «bons projets ou bonnes opportunités», permettent comme le relève Wirtz (2008a, p. 9) «d'élargir l'ensemble des 
options stratégiques à la disposition du dirigeant, grâce à une perception élargie de l'environnement (scanning) et à des modèles d'interprétation plus variés apportés par des administrateurs aux expériences hétérogènes. C'est donc un cas de renforcement de l'espace discrétionnaire grâce au levier cognitif et comportemental de la gouvernance». Cet élargissement des options stratégiques, ce soutien de l'actionnariat externe dans les instances de gouvernance nous paraissent constituer une vision crédible et explicative du caractère plus entrepreneurial (au sens de Covin et Slevin, 1989) ${ }^{14}$ des moyennes entreprises adossées, comme observé dans certaines investigations empiriques (Le Vigoureux, 1997).

Cela ne signifie pas la disparition du rôle disciplinaire des mécanismes de gouvernance dans la moyenne entreprise adossée. Cependant, avec cette approche, on peut d'autant mieux relativiser la portée des conflits d'agence, limités par la présence d'un actionnariat dominant et bien souvent par la solidité et le professionnalisme des investisseurs en capital (particulièrement les capital-risqueurs et les capital-développeurs) qui ont les compétences pour réduire l'asymétrie d'information mieux qu'un investisseur boursier quelconque.

Les approches cognitives de la gouvernance, en concevant l'entreprise comme un projet dynamique fondé sur la capacité créative d'une communauté d'acteurs de nature différente, alimentent ainsi de nouvelles réflexions sur le comportement humain au sein de l'organisation. En un sens, elles rejoignent la vision déjà ancienne mais en pleine redécouverte d'une auteure comme Mary Parker Follett qui prône le conflit constructif et intégratif où aucune partie prenante ne sacrifie ses intérêts ou ne compromet ses valeurs ${ }^{15}$. Il nous semble que ces approches se révèlent et s'expriment tout

14. Pour Covin et Slevin, sur la base d'une échelle de neuf items, un comportement stratégique entrepreneurial se caractérise par une innovation technologique et/ ou de produit importante, une orientation concurrentielle agressive et une forte propension au risque chez les dirigeants.

15. Pour Bessire,Chatelin et Onnée (2007, p. 20), «nous sommes ici à l'opposé de la vision traditionnelle de la gouvernance des organisations qui cherche à aligner les intérêts du dirigeant sur celui des actionnaires ou des parties prenantes et tente donc de résoudre le conflit, jugé néfaste, par au mieux le compromis, au pire la domination. Comme Follett, nous pensons que le conflit est une dimension intrinsèque et bénéfique de la dimension des organisations et qu'il faut, au lieu de le refouler, de renouveler sans fin les tentatives vouées à l'échec de le contenir, lui permettre de s'exprimer, sous peine de fossiliser l'entreprise: on sait les ravages que produit une culture d'entreprise trop forte et consensuelle: à terme la créativité, l'innovation sont étouffées». 
particulièrement au sein des moyennes entreprises adossées, où une coalition souvent familiale d'acteurs tend à renoncer au plein pouvoir au profit d'un projet productif revisité.

\section{Conclusion}

La moyenne entreprise, objet d'étude en tant que tel, peut constituer sur maints sujets un terrain propice aux constructions intellectuelles et aux explorations concrètes car elle offre un bon équilibre entre le nombre de variables à explorer et le caractère intelligible de leurs enchaînements. Comme le signale Desreumaux (2008, p. 86), «[...] il est évident que les plus grandes [entreprises], supposées en même temps les plus puissantes, ne doivent pas constituer le seul pôle d'intérêt. Il existe déjà, heureusement, des traditions de recherche sur les petites entreprises, les moyennes entreprises, les entreprises familiales, notamment nourries de travaux francophones, qu'il convient de maintenir voire de renforcer».

La moyenne entreprise, selon la nature et la structure de son actionnariat, développe des comportements stratégiques différenciés. Ceux-ci apparaissent entrepreneuriaux dans la forme adossée, point possible et fructueux de rencontre entre un actionnariat familial et des investisseurs externes. L'adossement, autrement dit l'ouverture partielle du capital, ne constitue pourtant pas une orientation naturelle des moyennes entreprises familiales indépendantes, au sein desquelles on peut souvent relever une certaine rationalité de la «non-croissance»: craintes d'éclatement du contrôle familial, de l'apparition de coûts d'agence, de rupture culturelle. Dans ce cadre, il est courant de voir des entreprises familiales, ayant épuisé les leviers de l'autofinancement puis de l'endettement, renoncer à de véritables opportunités de croissance pour éviter l'entrée de financeurs externes.

Au final, il nous semble que l'adossement n'entame ni le caractère ni l'identité familiale d'une moyenne entreprise. S'il introduit une relation d'agence, et donc des coûts, qui n'existait pas jusqu'alors, il prévient aussi le risque de sclérose familiale, l'altruisme inefficient, les coûts d'agence «intrafamiliaux ». Il ouvre de nouvelles perspectives de développement, professionnalise l'organisation, peut contribuer à rassurer les banquiers. L'approche cognitive de la gouvernance valorise d'ailleurs ces apports extérieurs comme autant de nouveaux leviers ou de réseaux en faveur de l'innovation en relativisant la dimension disciplinaire des dispositifs de gouvernance.

Lorsque l'entreprise est moins conçue comme un patrimoine que comme un projet, la propriété familiale est sans doute plus encline à l'adossement qui favorise alors le passage d'une pérennité de pouvoir, liée au contrôle du 
capital et des décisions à une pérennité de projet, qui correspond au maintien de l'activité principale et à l'essentiel de l'identité de l'organisation: «même si au fil du temps les activités changent, même si la direction n'est pas issue de ce même groupe, il reste un projet fédérateur de l'organisation, ciment de sa pérennité» (Mignon, 2001, p. 172).

\section{Bibliographie}

Allouche, J. et B. AmANn (1998), «La confiance, une explication aux performances des entreprises familiales », Economie et Société, série Sciences de gestion, vol. $25, \mathrm{n}^{\text {os }} 8-9$, p. 129-154. «Performances et caractéristiques financières comparées des entreprises familiales et non familiales : le rôle modérateur de la cotation en bourse et du degré de contrôle actionnarial», AIMS Montréal, XVI Conférence internationale de management stratégique.

AMIT, R., J. BRANDER et C. ZOTT (1998), «Why do venture capital firms exist ? Theory and Canadian evidence», Journal of Business Venturing, vol. 13, p. 441-466.

ANDERSON, R. et D. REEB (2003), «Founding-family ownership and firm performance: evidence from the S\&P $500 »$, Journal of Finance, vol. 58, n³ 3, p. 1301-1328.

ARRÈGLE, J.L. et I. MARI (2010), «Avantages ou désavantages des entreprises familiales », Revue française de gestion, vol. 36, n 200, p. 87-109.

ARrÈGLe, J.L., R. DURAND et P.VERY (2004), «Origine du capital social et avantages concurrentiels des firmes familiales », Management, vol. 7, nº 1, p. 13-36.

AurÉGAN, P., P. Joffre et F. LE VigOuREuX (1997), «Modèles d'analyse stratégique: contributions récentes », dans Encyclopédie de gestion, $2^{\mathrm{e}}$ édition, tome 2, Paris, Economica, p. 2041-2060.

BARCLAYS WEALTH INSIGHTS (2009), Family Business : in Safe Hands?, Étude rédigée par l'unité d'intelligence économique de Barclays Wealth, Londres.

BARTH, E., T. GULBRANDSEN et P. SCHONE (2005), «Family ownership and productivity: the role of owner-management », Journal of Corporate Finance, $\mathrm{n}^{\circ} 11$, p. 107-127.

BERGER, A.N. et G.F. UDELL (1998), «The economics of small business finance: the roles of private equity and debt markets in the financial growth cycle», Journal of Banking \& Finance, vol. 22, n $^{\text {os }}$ 6-8, p. 613-673.

Bessire, D., C. Chatelin et S. OnNÉE (2007), «Qu'est-ce qu'une bonne gouvernance?», XXVIII Congrès de l'Association francophone de comptabilité, Poitiers.

BRunninge, O., M. Nordqvist et J. WikLund (2007), «Corporate governance and strategic change in SMEs : the effects of ownership, board composition and top management teams », Small Business Economics, vol. 29, p. 295-308.

CALvi-REveYron, M. (2000), «Le capitalisme familial, dans un contexte français, induit-il moins de dividendes que les autres formes d'actionnariat?», Finance Contrôle et Stratégie, vol. 3, n¹, p. 81-116. 
CASTALDI, R. et M.S. WORTMAN (1984), «Boards of directors in small corporations: an untapped resource », American Journal of Small Business, vol. 9, n² 2, p. 1-10.

CENTRE D'ANALYSE STRATÉGIQUE (2009), «Les entreprises de taille intermédiaire: un potentiel d'innovation à développer? », La note de Veille, ${ }^{\circ} 131$.

CHARLIER, P. et G. LAMBERT (2008), «Entrepreneuriat familial en Europe et stratégie de gouvernance: une perspective positive des théories de l'agence», Journée de recherche entrepreneuriat et stratégie, Bordeaux.

CharreauX, G. (2002a), «L'actionnaire comme apporteur de ressources cognitives », Revue française de gestion, vol. 5, n 141, p. 77-107.

Charreaux, G. (2002b), «À la recherche de nouvelles fondations pour la finance et la gouvernance d'entreprise », Finance Contrôle Stratégie, vol. 5, n³, p. 5-68.

CharreauX, G. et P. WirTz (2006), Gouvernance des entreprises. Nouvelles perspectives, Paris, Economica.

Charreaux, G. (2008), «À la recherche du lien perdu entre caractéristiques des dirigeants et performance de la firme: gouvernance et latitude managériale», Cahier du Fargo, Université de Bourgogne, n 1080401, 42 pages.

CiÉPLY, S. (1995), «Le financement bancaire des PME. L'apport des modèles de coopération interentreprises ", II ${ }^{e}$ Congrès international francophone de la PME, Paris.

Cosier, R.A. et M. HARVEY (1998), «The hidden strengths in family business: functional conflict», Family Business Review, vol 11, n 1, p. 75-79.

COUdERC, J.P. et E. STEPHANY (2008), «La gouvernance d'entreprise a-t-elle un impact sur l'innovation?", Conférence internationale de la gouvernance d'entreprise, Bordeaux.

COVIN, J. et D. SLEVIN (1989), «Strategic management of small firms in hostile and benign environments », Strategic Management Journal, vol. 10, nº 1, p. 75-87.

DAILY, C. et D. DALTON (1992), «The relationship between governance structure and corporate performance in entrepreneurial firms », Journal of Business Venturing, vol. 7, p. 375-386.

DAVIS, J.H., F.D. SCHOORMAN et L. DonALDSON (1997), «Toward a stewardship theory of management », Academy of Management Journal, vol. 22, n¹, p. 20-47.

DePRET, M.H. et A. HAMdOUCH (2005), «Gouvernement d'entreprise et performance », dans Gouvernement d'entreprise: Essai de synthèse et nouvelles voies de recherche, Bruxelles, De Boeck, p. 39-79.

Desreumaux, A. (2008), «Refaire de la stratégie?», Finance Contrôle Stratégie, vol. 11, hors série, p. 67-107.

DONCKELS, R. et E. FROHLICH (1991), «Are family business really different? European experiences from STRATOS », Family Business Review, vol. 4, n² 2, p. 149-160.

FERNANDEZ, C.M. et M. COBAS (1998), «Effets indirects des sociétés de capital-risque dans les $\mathrm{PME}$, Congrès international francophone de la PME, Lille. 
Forbes, D.P. et F. Milliken (1999), «Cognition and corporate governance: understanding boards of directors as strategic decision-making groups », Academy of Management Review, vol. 24, n³, p. 489-505.

FuEglistaller, U. et T.M. ZELLWEGER (2006), Rentabilité et règles du jeu des entreprises familiales, Université de Saint-Gall, Ernst \& Young SA, Zurich.

GABRIELSSON, J. et M. Huse (2002), «The venture capitalist and the board of directors in SMEs: roles and processes », Venture Capital, vol. 4, n 2, p. 125-146.

GAllo, M.A. et A. VilasecA (1998), «An empirical examination of ownership structure in family and professionally managed firms », Family Business Review, vol. $5, \mathrm{n}^{\circ} 2$, p. $117-136$.

GomeZ-MeJiA, L., M. NunEZ-NiCKEL et I. GuTIERREZ (2001), «The role of family ties in agency contracts », Academy of Management Journal, vol. 44, n 1, p. 81-95.

GREPME (1994), Les PME. Bilan et perspectives, Paris, Economica.

GUÉRY-STEVENOT,A. (2006), «Conflits entre investisseurs et dirigeants. Une analyse en termes de gouvernance cognitive», Revue française de gestion, vol. 5, $\mathrm{n}^{\circ} 164$, p. 157-180.

HABBERSHON, T. et M. WiLliams (1999), «A resource-based framework for assessing the strategic advantages of family firms », Family Business Review, vol. 12, n 1 , p. 1-24.

Hirigoyen, G. (1984), Contribution à la connaissance des comportements financiers des moyennes entreprises industrielles et familiales, Thèse de l'Université de Bordeaux I.

Hirigoyen, G. (2002), «Le gouvernement des entreprises familiales », dans La gestion des entreprises familiales, sous la direction de G. Hirigoyen et J. Caby, Paris, Economica, p. 17-30.

Hirigoyen, G. (2008), «Biais comportementaux dans l'entreprise familiale : antécédents et impacts », Revue Économie et Sociétés, Série Économie de l'entreprise, vol. 10, n 19 , p. 1901-1930.

HiRigoyen, G. (2009), «Concilier finance et management dans les entreprises familiales », Revue française de gestion, $\mathrm{n}^{\text {os}} 198-199$, p. 393-411.

KENYON-RouvineZ, D. et J.L. WARD (2004), Les entreprises familiales, Paris, Presses universitaires de France, coll. «Que sais-je?».

Kets DE VRIES, M. (1988), «The dark side of CEO succession», Harvard Business Review, vol. 63, n 6, p. 56-60.

KPMG (2007), L'entreprise familiale, une entreprise décidément pas comme les autres, Étude interne.

LE VigOUREUX, F. (1997), «Entreprises moyennes: structures de propriété et comportement stratégique», Revue française de gestion, p. 71-84.

LOFTI, K. et K. WAFA (2007), «Formes d'activation des conseils d'administration dans les PME. Une étude exploratoire dans le contexte français», $X V I^{\mathrm{e}}$ Conférence internationale de management stratégique, Montréal, 6-9 juin. 
MARANDON, G. (2003), «Au-delà de l'empathie, cultiver la confiance: clés pour la rencontre interculturelle», Revista CIDOB d'Afers Internacionals, mai-juin, $\mathrm{n}^{\text {os }} 61-62$, p. 259-282.

Mignon, S. (2000), «La pérennité des entreprises familiales: un modèle alternatif à la création de valeur pour l'actionnaire », Finance Contrôle Stratégie, vol. 3, $\mathrm{n}^{\mathrm{o}} 1$, p. 169-196.

Mignon, S. (2001), Stratégie et pérennité de l'entreprise, Paris, Vuibert.

Mignon, S. (2009), «La pérennité organisationnelle», Revue française de gestion, vol. 2, n 192, p. 73-89.

Miller, D., I. LE BRETON-Miller et B. SCHOLNICK (2008), «Stewarship vs. Stagnation: an empirical comparison of small family and non-family businesses », Journal of Management Studies, vol. 45, n¹, janvier, p. 51-78.

MorCK, R., A. SHLEIFER et R. VishnY (1988), «Management ownership and market valuation», Journal of Financial Economics, vol. 20, p. 293-315.

Mustakallio, M., E. Autio et S. ZAHRA (2002), « Relational and contractual governance in family firms: effects on strategic decision-making », Family Business Review, vol. 15, n³, p. 205-222.

MYERS, S. (1984), «The capital structure puzzle», Journal of Finance, vol. 39, n 3, p. $575-592$.

NALDI, L. et M. NORDQVIST (2008), Family Firms Venturing into International Markets: A Resource Dependence Perspective, Washington, SBA Office of Advocacy, juin.

Neubauer, F. et A. LANK (1998), The Family Business. Its governance for Sustainability, Londres, McMillan Business.

NorTOn, E. (1991), «Capital structure and small public firms», Journal of Small Business, vol. 1, n², p. 161-177.

Pouget, J. et E. STEPHANy (2002), «Gouvernance de la relation capital-risqueurentrepreneur», VI ${ }^{e}$ Congrès international francophone de la PME, HEC Montréal.

POUTZIOURIS, P.Z. (2001), "The views of family companies on venture capital: empirical evidence from the UK small to medium-size enterprising economy », Family Business Review, vol. 14, n³ , p. 277-291.

PRICEWATERHOUSECOOPERS (2006), Enquête sur les entreprises familiales françaises, publication interne.

RETAILlEAU, B. (2010), Les entreprises de taille intermédiaire au cour d'une nouvelle dynamique de croissance, février, rapport confié par le Premier ministre au sénateur Bruno Retailleau, France.

SCHulze, W.S., M. LubATKIN et R. DinO (2001), «Agency relationships in family firms: theory and evidence», Organization Science, vol. 12, n², p. 99-116.

SCHUlZE, W.S., M. LUBATKIN et R. Dino (2002), «Altruism, agency and the competitiveness of family firms Management», Decision Economic, vol. 23, $\mathrm{n}^{\text {os }} 4-5$, p. 247-259. 
Sharma, P., J.J. Chrisman et J.H. ChUA (1997), «Strategic management of the family business : past research and future challenges », Family Business Review, vol. $10, \mathrm{n}^{\mathrm{o}} 1, \mathrm{p} .1-35$.

SIRMON, G.D. et M.A. HITT (2003), «Managing resources: linking unique resources, management, and wealth creation in family firms », Entrepreneurship Theory and Practice, vol. 27, $\mathrm{n}^{\circ}$ 4, p. 339-358.

STEPHANy,E. (2001), «L'évolution des pratiques du capital-risque en France», Revue française de gestion, $\mathrm{n}^{\circ} 135$, p. 63-75.

Stephany, E. (2003), La relation capital-risque PME, Bruxelles, De Boeck, coll. «Finance, comptabilité contrôle».

WiRTZ, P. (2008a), «Les firmes entrepreneuriales en croissance ont-elles un système de gouvernance spécifique?», Cahier du Fargo, Université de Bourgogne, $\mathrm{n}^{\mathrm{o}} 1080701,31$ pages.

WIRTZ, P. (2008b), Les meilleures pratiques de gouvernance d'entreprises, Paris, La Découverte, coll. «Repères ».

Zellweger, T.M., K.A. Eddleston et F.W. Kellermanns (2010), «Exploring the concept of familiness : introducing family firm identity », Journal of Family Business Strategy, vol. 1, p. 54-63. 